\title{
Comparative Analysis of Deep Learning Methods for Detection of Keratoconus
} Impana N1, K J Bhoomika2, Suraksha S S3, Karan Sawhney4, Priya D5, Mamatha G S6

\section{1-4Dept. Of Information Science and Engineering, RV College of Engineering, Bengaluru, Karnataka,} India

\section{Associate Professor, Dept. Of Information Science and Engineering, RV College of Engineering, Bengaluru, Karnataka, India}

\begin{abstract}
Keratoconus eye disease is not an inflammatory corneal disease that is caused by progress in thinning of the cornea, scarring, and deformation in the shape of the cornea. In India there is a significant increase in the number of cases of the keratoconus, and several research centres have been paying attention to this disease in recent years. In this situation, there is an immediate need for tools that simplify both diagnosis and treatment[1]. The algorithm developed can decide whether the eye is normal eye or keratoconus eye with stages. The K-net model analyzes the pentacam images of eye using a convolutional neural network $(\mathrm{CNN})$ a deep learning model and pre trained ResNet-50 and InceptionV3 pre trained models and does the comparitive analysis of the accuracies of these models. The results show that the Keratoconus Detection algorithm leads to a good job, with a 93.75 per cent accuracy on the data test collection. Keratoconus Detection model is a program that can help ophthalmologists test their patients faster, therefore reducing diagnostic errors and facilitating treatment.
\end{abstract}

Key Words: Deep learning, Convolution Neural Network (CNN), Keratoconus detection, corneal topography.

\section{INTRODUCTION}

Keratoconus is a condition where the cornea of the eye starts getting thinner in progress. The cornea is nothing but the outer layer of the eye in the front[1]. Patients having this keratoconus eye disease will have a coneshaped cornea. Once the cornea starts thinning and having a sharp inclination, the surface in the front will begin becoming irregular in shape over specific progress. Because of all these, the patients can experience blurred visions.

The thickest part of the cornea is its middle layer, which is mainly made up of the protein known as collagen and water.[1][3]

This protein called collagen makes the cornea solid and elastic, which helps keep the regular round shape.
A fine cornea will be able to focus the light, hence gives a clear vision. Whereas in the keratoconus eye, the light gets refracted, which can cause blur in vision or loss of sight.

The cornea's tissue is made up of five layers. The first layer is the epithelium[5]. Each layer of this can presume in keratoconus. The epithelial cells can be stretched and zoomed in. This keratoconus disease can be caused by having a family history, age, rubbing of the eyes, allergies, etc. Corneal topography is used in detecting the keratoconus eye.

In any case, several patients having hypersensitivities tend to wipe their eyes unnecessarily, which can cause the thinning of the cornea. There has been various kind of methods which were proposed in correcting this condition. Techniques like rigid RGP lenses, scleral lenses, and other types of surgeries were performed to correct this state. If you consider India, the research done on this keratoconus eye disease is significantly more minor. Many researchers are trying to find information about the condition and the right treatments for the same. This can develop in people of any age group and, if not treated early, can lead to blindness[11]. It is mainly treated glasses, lenses in the early stage of this disease.

There are four groups of the keratoconus disease, namely the Forme Fruste, Nipple Cone, Oval Cone, and the Globus Cone. The stages of this keratoconus eye detected here will be stages 1,2,3,4, respectively.

The most-mild form of the keratoconus stage is the Forme Fruste. It does not show any kind of symptom and is subclinical.

Corneal Topography is the only possible way to detect this stage. Sometimes in this stage, the disease will start, and suddenly, it will stop progressing. Treatment is not necessary for this stage.

The second stage, which is the Nipple Cone, is the one that usually affects a little area of the cornea at the centre. Even though the area affected is less, it will still cause some alteration in the eye's vision.

Sagging or the Oval Cone is the third stage of keratoconus. 
In this, the area affected is more significant in size than that of the nipple cone. It is usually seen towards the ear on the cornea. It looks like there is a sag happening in the cornea hence the name. The bulging and thinning of the cornea area is in the direction down on the cornea, and the deform carried out can be extended to the center parts of the cornea[2].

The last stage is the Globus Cone stage. In this stage, the cornea is affected by above $75 \%$ of the area. This is one of the advanced stages of keratoconus eye disease. It cannot be treated with glasses, and hence it may require corneal transplantation to be done.

The corneal transplant will be required in most severe cases, which will have added risks like infection and sometimes blindness. Therefore, it is advised to have a proper diagnosis whenever possible before the progression of the disease takes place.

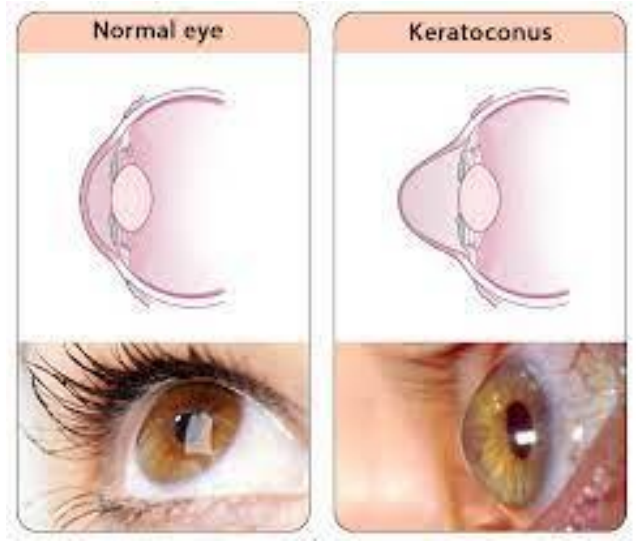

Fig 1: Normal eye and keratoconus eye cornea

Consistent advancement of enormous arrangements of ophthalmic information, supported by the progress of learning calculations, and the expanded handling power have prompted increased revenue in using deep learning or machine learning methods[15]. Deep learning is helping researchers in analyzing medical images in different fields and, it has reported very good performance. The convolution neural network (CNN) gives high accuracy and from more training data the layered hierarchical image features will be learnt.

The exact goal of this particular research paper is that it can decide whether an eye suffers from keratoconus or not and, if yes, then which stage it is present in[11]. Fig 1. shows the differences between an ordinary cornea and one affected with keratoconus infection.

\section{STUDY ON RELATED WORK}

Recently, deep learning techniques have made a significant contribution to the detection, or the detection of a variety of diseases in the healthcare system, and has a lot of benefits, such as detection of insurance fraud, which is to provide medical care to the patients and at a fair price, to discover better methods of treatment, the creation of innovative medical programs, the success of the treatment, healthcare, infrastructure, hospitals, infection control, and the improvement of patient care, as well as the retention, better customer relationship management. Diagnose illnesses, and it is an important area in the fields of science, especially at a time like this. Under the present lifestyle of people, labour, climate, diet, diseases that have been created, one of which is the eye disease Keratoconus.

This is the first version of an experimental study that uses a variety of deep learning methods for treating keratoconus.

Alexandru Lavric, and Popa Valentin[3] in 2019 introduced a method where the corneal geography of the eye is detected using CNN model which was able to learn and extract features of an affected eye.

R. Kanimozhi and Dr. R. Gayathri[6] in 2019 published a paper where they used the algorithms and predictive models to predict if the disease is present or not. The paper discussed new ways for the early stage detection of keratoconus.

P. Agostino Accardoa, and Stefano Pensierob[12] in 2019 published a paper on their research on keratoconus using

convolution neural networks. This way of detection was purely on smartphones. Smartphones were used to detect images and it gave them an accuracy of about $72.9 \%$ as a whole and different percentages for different stages of the detection.

Francisco J. F. Cañavate[11] and Jorge L. Alio worked on detecting keratoconus based on new corneal volumetric analysis. The work used raw topographic data and on using that it performed morphometric modeling of the cornea with the aid of CAD.

Ariela Gordon-Shaag, Michel Millodot, and Einat Shneor[10]-Published a paper on the generic and environmental factors of keratoconus. The paper will give brief overview of the researches done in the recent times on the development of keratoconus epidemiology and genetic subjects. The causes are not just the rubbing of eye, sunlight, etc, but also the other environmental factors.

\section{Amira Soltani, TaharBattikh, ImedJabri[15]}

Of late, clinical imaging has developed as it addresses a fundamental help for the patients' analysis and observing. Early acknowledgment of illnesses, for 
example, "Keratoconus" is principal to the supposition. The achievement chance is high for the treatment gave previously. Since the state of the optic nerve's exhuming is the main element in its ID, right location methods are important to feature their structures. This paper presents a similar investigation of various edge location's strategies applied on a bunch of ophthalmologic pictures of the optic nerve.

Shivangi C. Patel, Manish I. Patel in 2018 did a research called the analysis of CDR of keratoconus images for the disease detection. Since keratoconus is one of the leading eye disease causing blindness and no early symptoms detected they used CDR method. Disc ratio (CDR) was used to categorize if the eye was an healthy eye or keratoconus affected. In this method the disease is detected using retinal image. In this paper, further analysis of CDR is done by using different color channel combinations of the keratoconus images.

\section{DATASET AND PREPROCESSING}

sDataset collected for the research is from some of the hospitals across Bengaluru. There are about 430 images collected. The images show the corneal topographies on an eye. The device used to scan is called the pentacam. Pentacam images or the scans done show the exact measure for the front and back of the cornea.

In the datset there are five categories that is normal Fig2 B, Forme Fruste Fig3A, Nipple ConeFig3B,Oval ConeFig3C and Globus ConeFig3D

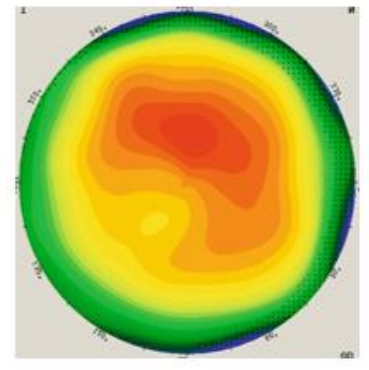

Fig 2. A) Keratoconus affected

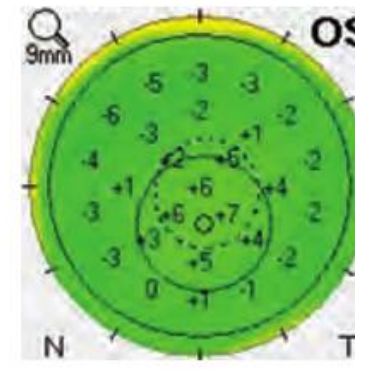

B) Normal eye

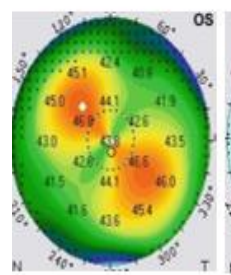

t. A) Forme Fruste

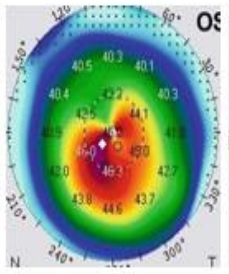

B) Nipple Cone

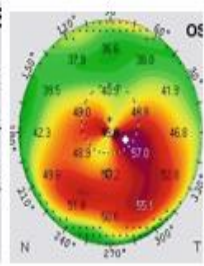

C) Oval Cone

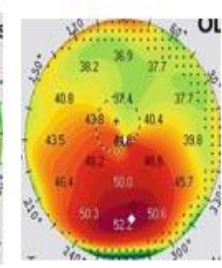

D) Globus Cone
Fig 3. Stages of keratoconus
The dataset is split into training and testing dataset, about 430 images for training and 50 images for testing. Preprocessing of image pixels into uniform scale of $150 \mathrm{X} 150$ to make faster convergence. Binary classification- process is applied to distinguish the keratoconus dataset into two classes only. It will immediately classify if the image which is analyzed is a normal eye or a keratoconus affected eye. Data agumentation is performed to improve the performance by forming new and different examples to train datasets, rescaling the image pixel in range [0,255]$>[0,1]$, rotation, flipping, shearing. Training and testing data set target size will be 150X150.

Multi-level classification- In this process, the images identified as the disease one will further be classified into stages as seen in Fig 3.

\section{DESIGN OF THE SYSTEM}

Model is trained using CNN, ResNet50, and InceptionV3, and then the classification will take place. The next step is followed by the comparison of the test image and trained model by displaying the result. In the case of keratoconus, it will be classified as different stages.

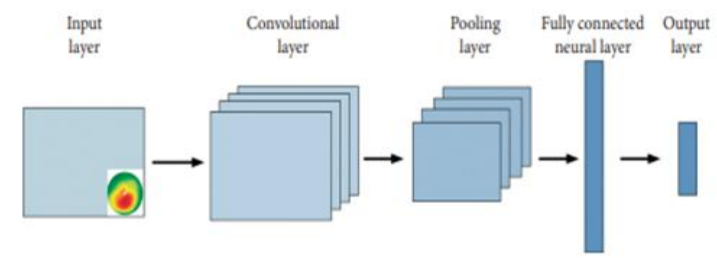

Fig 4. System Architecture

\section{K-net model}

The fundamental objective is to execute and test a calculation that permits keratoconus discovery by working with the emotional cycle. The analysis is performed using a convolution neural network. The most utilized methodology used to analyze and confirm keratoconus is to create corneal geology, which is later deciphered by the ophthalmologist trained professional.

The pictures will think about the contribution of Keratoconus Detection calculation inside the learning interaction related to the convolutional neural networks $(\mathrm{CNN})$. The neural pixels created to measure the collected information (e.g., pixel upsides of a picture addressing the corneal geography) utilizing loads on associations among the neurons. The learning cycle includes the continuous change of these loads to lessen the blunder in both classification and to get to know the measures. 
The K-net utilizes a filter with a size of $3 \times 3$ means of three pixels for analyzing the method of the preparation. 32 neurons are applied on the convolution layer(example the number of filters)that companion with some things very comparable locale of the informational data index.

A cushioning highlight is utilized to make the highlights map at the neural network layer. For the state of affairs (a filter of three), a cushioning of one is applied to guarantee that the spatial yield size is equivalent to the info length. The following coordinated layer is the standardization layer that allows the advancement of the agency by adjusting.

There is another layer trailed through a relu layer. Relu is a rectified direct unit layer. The motivation in the back of this accretion is to add nonlinearity after each convolutional layer. This layer brings down the education season of the enterprise and diminishes the affectability of K-net.

The subsequent layers of the planned K-net is a pinnacle pooling layer that has the part of creating a downsampling interest to put off repetitive statistics from the layers. This cycle is applied to assemble the range of filters without the strengthening growth in pressure. The most pooling layer returns the most excessive upsides of the defined square locales, utilizing a step combined with a proper step duration of two for the steerage interplay.

The following diploma is to construct the number of filters from 32 to 128 and play out the standardization duties accompanied utilizing the use of the relu layer. After those sports have been fulfilled, the most pooling ability is applied. The statistics are pre-had once more, this time by way of a sixty four-neuron convolutional layer accompanied with the aid of standardization and a relu layer.

The final one is a very associated layer wherein every one of the neurons interface with the neurons from the past layers and change records. This deposit joins each one of the highlights eliminated and observed through manner of the beyond layers as to differentiate designs within the facts.

On this residue, every one of the separated highlights are utilized to installation the corneal geography. Time taken during this is same as the classification lessons in the case of the keratoconus eyes and one for typical ones.

The last layer used is called the softmax layer, which is used for multiclass image classification. The magnificence layer assigns one of the training to every input and calculates the loss parameter the usage of an activation feature.[20]
Many learning calculations use multi-facet networks between data sources and yields. These neural organizations permit the identification of highlights, examples, and qualities inside the classification relationship. Innovative advancement has prompted the turn of events and clarifying these calculations, with it being utilized in numerous spaces in medication with trust. One of the principal techniques for perceiving and ordering pictures is a CNN in neural network. CNN's are at present utilized in applications, for example, object acknowledgment and face recognition. A convolution neural network that is equipped for the detection of the keratoconus infection is shown in this paper. Convolution and pooling layer 4, 2 fully connected layers and output layer are present.

K-net model is built in this method by training and testing the dataset and the accuracy obtained by this is around $93.75 \%$. Fig 6 represents the architecture of K-net model.

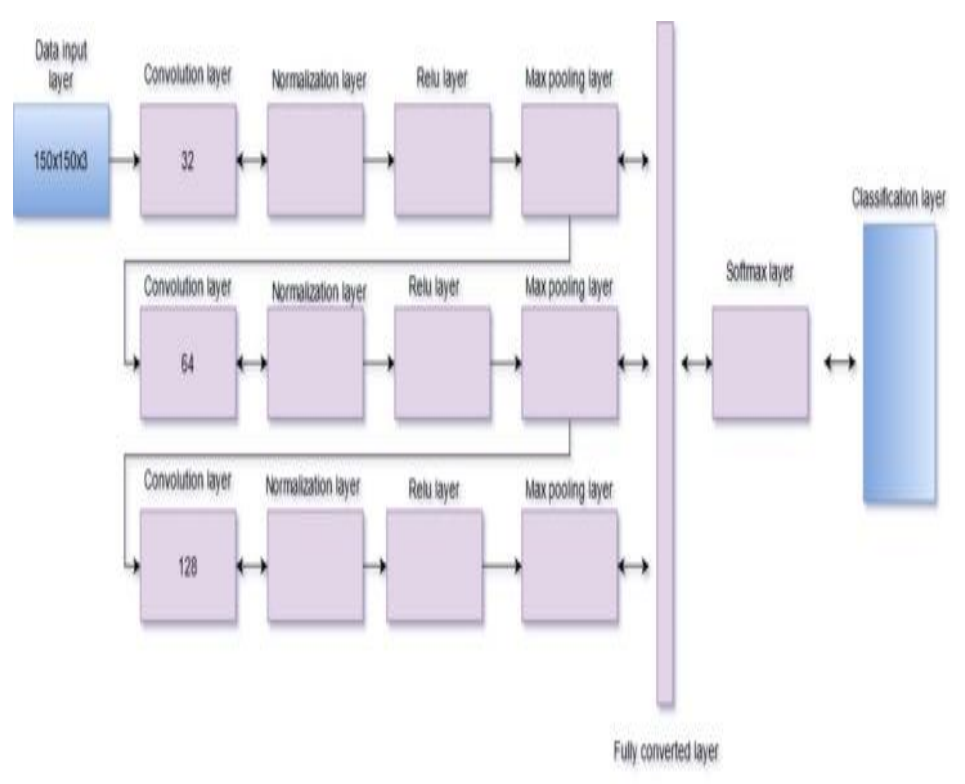

Fig 5. K-net Architechture

\section{ResNet50 model}

ResNet50 also called the Residual Networks is one the transfer learning model used. Transfer models are pretrained on a dataset called ImageNet[17]. Fig 6 shows the architecture of the ResNet50 model. The testing accuracy obtained by this is $93 \%$. 


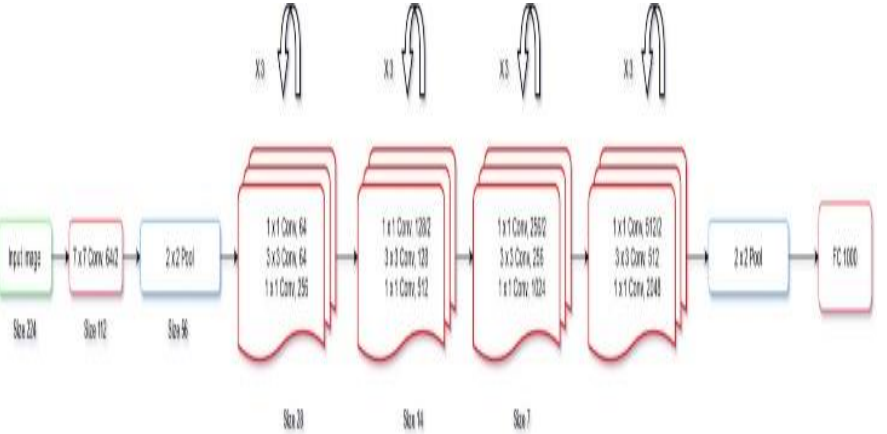

Fig 6. ResNet50 Architecture

\section{Inceptionv3 model}

Inceptionv 3 is another pre-trained model that is used in this paper. Fig 7 represents the architecture of the Inceptionv3 model. Multi-class image classification is done using these models and the accuracy obtained by this in the test set is around $94 \%$

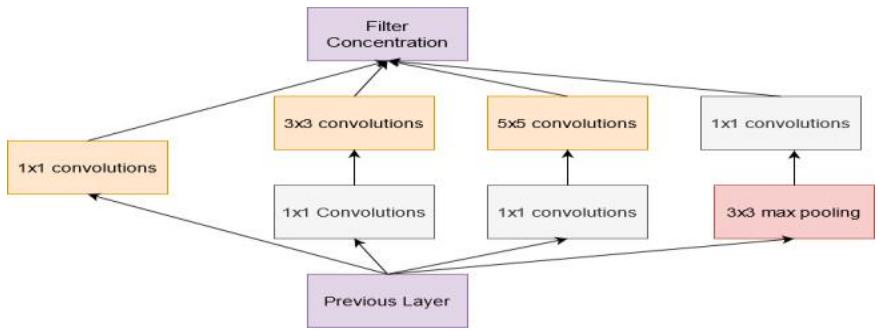

Fig 7. Inceptionv3 Architecture

\section{RESULTS AND DISCUSSION}

The automated image detection system has made a significant change in the detection of the disease by saving the effort and time and charge of the ophthalmologists and the patients. Early-stage detection is the most important step in the keratoconus condition.

In Figure 8 we are getting the accuracy parameter while using 50 epochs or iterations. Every change estimates the variation, which will correspond to updates of the neural system. The algorithm we are using has an accuracy obtained around $93.75 \%$ for the statistics, which consists of about 435 pentagram pictures. The accuracy validated is taken for every 50 iterations. There is an accuracy parameter, and loss parameter analyzed.

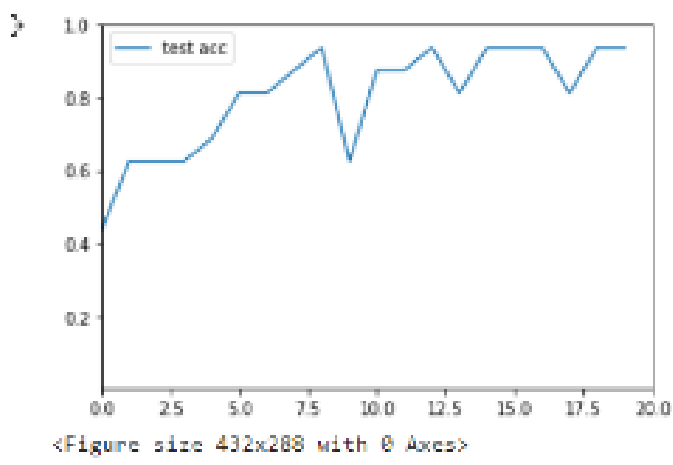

Fig 8. Accuracy parameter

The CNN's loss parameter is proven in Figure 7. The pass entropy of the complete $\mathrm{CNN}$ is represented via this parameter. When the community's accuracy is progressed, the loss parameter decreases substantially, ensuing in the removal of inaccurate topography type.

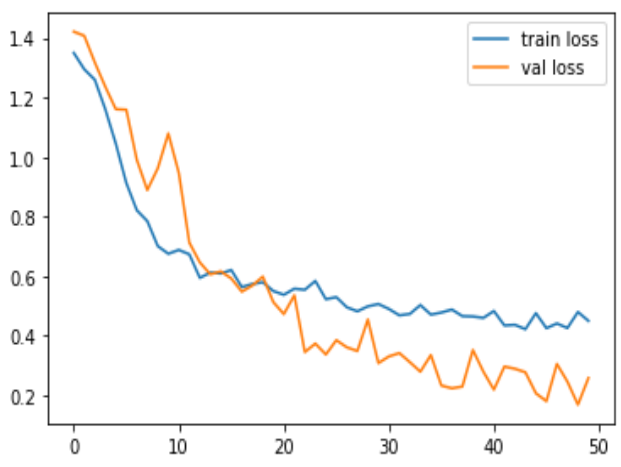

Fig 9. Loss parameter

\section{Mathematical notations}

Conv2D layers present in the K-net model are 4 and the method of calculating the parameters for the layers is shown ahead in the formulas. The bias which is connected with every filter is represented as 1 .

The learning rate used is 0.0001 which is the alpha and is a constant.

Dimension of the image can be represented in a mathematical way as

$$
\operatorname{Dimsn}(\mathrm{img})=(\mathrm{nH}, \mathrm{nW}, \mathrm{nC})
$$

$\mathrm{nH}$ - height

$\mathrm{nW}$ - width

$\mathrm{nC}$ - number of channels 
In the K-net model, the image used is an RGB image and so the $\mathrm{nC}=3$ as we have Red, Green and Blue. In agreement with that, consider filter or kernel $\mathrm{K}$ is to be squared and have an odd dimension noted as $\mathrm{f}$. The dimension of filter is given as:

$$
\text { Dimsn(filter })=(\mathrm{f}, \mathrm{f}, \mathrm{nC})
$$

The filter used in this model in the convolution layer is a two dimensional matrix which is the convolution product attained between image and filter and is represented as:

$$
\operatorname{conv}(I, K)_{x, y}=\sum_{i=1}^{n_{H}} \sum_{j=1}^{n_{W}} \sum_{k=1}^{n_{C}} K_{i, j, k} I_{x+i-1, y+j-1, k}
$$

When the downsampling step is used, the elements of the image's are summed up to the information. Through every channel the activity is carried out and hence affects only the height and width(nH, nW) dimension keeping channel constant(nC).

$\operatorname{dim}($ pooling $($ image $))=\left(\left\lfloor\frac{n_{H}+2 p-f}{s}+1\right\rfloor,\left\lfloor\frac{n_{W}+2 p-f}{s}+1\right\rfloor, \mathbf{n}_{\mathbf{C}}\right) ; s>0$
$=\left(n_{H}+2 p-f, n_{W}+2 p-f, \mathbf{n}_{\mathbf{C}}\right) ; s=0$

Considered square filter size $\mathrm{f}$ is set to $\mathrm{f}=2$ and $\mathrm{s}=2$.

Max pooling is used in the model and in this the maximum element in the filter is retuned.

Activation function is denoted by $\psi$. First layer we represent:

$a^{[l-1]}$ with size $\left(n_{H}^{[l-1]}, n_{W}^{[l-1]}, n_{C}^{[l-1]}\right), a^{[0]}$ being the image in the input

Input layer: $\quad a^{[l]}$ with size $\left(n_{H}^{[l]}, n_{W}^{[l]}, n_{C}^{[l]}\right)$

Padding: $\quad p^{[l]}$ stride: $\quad s^{[l]}$

Filter numbers:

$n_{C}^{[l]}$ where each $K^{(n)}$ has the dimension: $\left(f^{[l]}, f^{[l]}, n_{C}^{[l-1]}\right)$

Nth convolution bias : $b_{n}^{[l]}$ Activation : $\psi^{[l]}$

Output layer:

$$
\begin{gathered}
n_{H / W}^{[l]}=\left\lfloor\frac{n_{H / W}^{[l-1]}+2 p^{[l]}-f^{l l]}}{s^{l l}}+1\right\rfloor ; s>0 \\
=n_{H / W}^{[l-1]}+2 p^{[l]}-f^{[l]} ; s=0 \\
n_{C}^{[l]}=\text { number of filters }
\end{gathered}
$$

Thus, this is the obtained mathematical notation for the first layer.

In fully connected layer, considering jth node and ith layer we show

$$
\begin{gathered}
z_{j}^{[i]}=\sum_{l=1}^{n_{i-1}} w_{j, l}^{[i]} a_{l}^{[i-1]}+b_{j}^{[i]} \\
\rightarrow a_{j}^{[i]}=\psi^{[i]}\left(z_{j}^{[i]}\right)
\end{gathered}
$$

$\mathrm{Wj}, 1$ represents the weights with parameters.

All the above represent the mathematical notations of the cnn model built.

After building and training the models for the dataset, the analysis of testing accuracy and training accuracy is done.It compares the accuracies with the transfer learning methods of ResNet50 and InceptionV3.

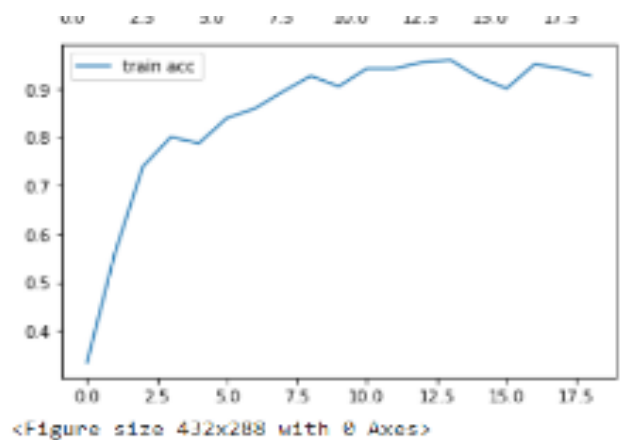

Fig 10. ResNet50 accuracy

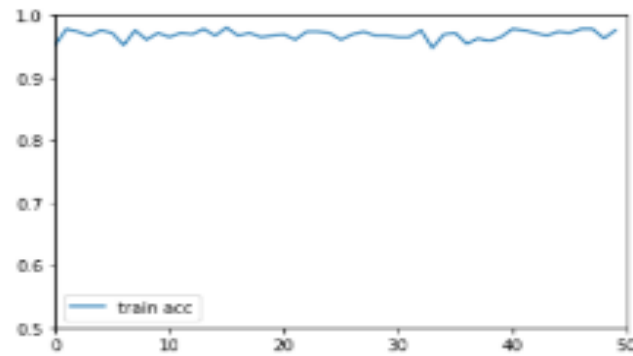

Fig 11. Inceptionv3 accuracy 
Table 1 represents a comparison between the three models with their accuracies.

\begin{tabular}{|l|l|}
\hline \multicolumn{1}{|c|}{ Model } & Testing accuracy \\
\hline K-net & $93.75 \%$ \\
\hline ResNet50 & $93.75 \%$ \\
\hline
\end{tabular}

Table 1. Comparing the accuracies of the given three models

\section{CONCLUSION}

The principle gain of this analysis is that it thoroughly may be applied as an incorporated piece of the symptomatic interplay. From the received results, we can propose that the proposed Keratoconus Detection calculation ensures an undeniable diploma of execution[26]. The principle dedication of this work is the flip of events and coordination in the analytic interplay of companion programming to help the ophthalmologist. AI calculations can probably intervene on vintage fashion scientific screening packages, having the option to provide diagnostics in a swift time body just as assisting with increasing affected person attention and solace.

The commitment of this paper comprises making use of a deep getting to know element to keratoconus contamination identification. This is to make prediction and execution easy for the doctors to diagnose and treat this keratoconus in a more efficient way. Similarly, after the ophthalmological convention, the corneal topography is applied as a contribution to the all-around organized neural organization, and this could determine if the patient is suffering from keratoconus or now not. By advancing the limits related to the convolutional neural community, the precision of the proposed calculation changed into accelerated to $93.75 \%$ for the take a look at the set.

The accomplished analysis measures practically corneal topologies and classifies them into classifications, recognizing designs related to the condition. In this paper, depending on the corneal topography, the analysis is done by mastering the calculations and screening tools. The analysis may be carried out inside the machine to help the eye doctors analyze the patient's conditions using geography [9]. The algorithm may be added to the instrument that conducts topography to help the ophthalmologist display screen patients greater speedy. Although the outcomes reveal a greater degree of percentage, the mechanism used should not be considered a stand-on own prognosis technique, but alternatively as a complement to the ophthalmologist's analysis of different scientific facts, including blood stress and corneal form evolution.

These algorithms will grow more incredibly green within the destiny, considering less complicated keratoconus detection and a discount in corneal transplant instances.

\section{References}

[1] Juan Carrillo, Lola Bautista, "KERATOCONUS DETECTION USING KERATOCONUS IMAGES OF THE EYE", 2019 XXII Symposium on Image, Signal Processing and Artificial Vision (STSIVA).

[2] Shivangi C. Patel, Manish I. Patel, "Analysis of CDR of Keratoconus Images for Keratoconus Detection," Proceedings of the 2nd International Conference on Trends in Electronics and Informatics (ICOEI 2018).

[3] Alexandru Lavric, and Popa Valentin in 2019,25 October 2018; Revised 19 November 2018; Accepted 24 December 2018; Published 23 January 2019, 9pages.

[4] Khan, Fauzia, et al. "Detection of Keratoconus using retinal Keratoconus images", Biomedical Engineering International Conference (BMEiCON), 6th. IEEE, 2013.

[5] S. A. Hasan and M. Singh, "An algorithm to differentiate astigmatism from Keratoconus in Axial Topographic images," 2015 International Conference on Industrial Instrumentation and Control (ICIC), Pune, India, 2015, pp. 1134-1139, DOI: 10.1109/IIC.2015.7150918.

[6] Ramaswamy, Kanimozhi \& Rajaraman, Gayathri. A Survey on Detection of Keratoconus 2018 International Journal of Scientific Research in Science,pp 304-9 .

[7] B. Askarian, F. Tabei, G. A. Tipton and J. W. Chong, "Novel Keratoconus Detection Method Using Smartphone," 2019 IEEE Healthcare Innovations and Point of Care Technologies, (HI-POCT), Bethesda, MD, USA, 2019, pp. 60-62, DOI: 10.1109/HIPOCT45284.2019.8962648.

[8] B. Askarian, F. Tabei, G. A. Tipton and J. W. Chong, "Novel Keratoconus Detection Method Using Smartphone," 2019 IEEE

Healthcare Innovations and Point of Care Technologies, (HI-POCT), 
Bethesda, MD, USA, 2019, pp. 60-62, DOI: 10.1109/HIPOCT45284.2019.8962648.

[9] M. Ucar, B. Sen and H. B. Cakmak, "A novel classification and estimation approach for detecting keratoconus disease with intelligent systems," 2013 8th International Conference on Electrical and Electronics Engineering (ELECO), Bursa, Turkey,2013,pp.521525,DOI:10.1109/ELECO.2013.6713897.[10] Gordon-Shaag, Ariela \& Millodot, Michel \& Shneor, Einat \& Liu, Yutao. (2015). The Genetic and Environmental Factors for Keratoconus. BioMed Research International. 2015. 10.1155/2015/795738.

[11] Saini JS, Saroha V, Singh P, Sukhija JS, Jain AK. Keratoconus in Asian eyes at a tertiary eye care facility Clin Exp Optom. 2004;87:97-101.

[12] Rostami, S. Detecting Keratoconus Using Convolutional Neural Network on Smartphone. Preprints 2020, 2020040271 (doi: 10.20944/preprints20200

[13] R. Manjula Sri, M. Raghupathi Reddy, K.M.M. Rao, "Hardware Implementation of Detection of Keratoconus from Color Keratoconus Images", pp. 340-345.

[14] AmslerM. Some of the data on the problem of the keratoconus Bull sot belgee opthamal.1961;129;331-54

[15] Amira Soltani, TaharBattikh, ImedJabri. Imagenet classification with the deep convolutional neural networks. Advances in Neural Information Processing Systems. 2012;1097-1105.

[16]Valdés-Mas, M.A., et al.: A new approach based on Machine Learning for predicting corneal curvature (K1) and astigmatism in patients with keratoconus after intracorneal ring implantation. Comput. Methods Programs Biomed. 116, 39-47 (2014).

[17]Souza, M.B., Medeiros, F.W., Souza, D.B., Garcia, R., Alves, M.R.: Evaluation of machine learning classifiers in keratoconus detection from orbscan II examinations. Clinics 65, 1223-1228 (2010).

[18] Arbelaez, M.C., Versaci, F., Vestri, G., Barboni, P., Savini, G.: Use of a support vector machine for keratoconus and subclinical keratoconus detection by topographic and tomographic data. Ophthalmology 119, 2231-2238 (2012).

[19] Ali, A.H., Ghaeb, N.H., Musa, Z.M.: Support vector machine for keratoconus detection by using topographic maps with the help of image processing techniques. IOSR J. Pharm. Biol. Sci. (IOSR-JPBS) 12(6), 50-58 (2017). Ver. VI. eISSN:2278-3008. p-ISSN:2319-7676.

[20] Kovács, I., et al.: Accuracy of machine learning classifiers using bilateral data from a Scheimpflug camera for identifying eyes with preclinical signs of keratoconus. J. Cataract Refract. Surg. 42, 275-283 (2016).
[21]Toutounchian, F., Shanbehzadeh, J., Khanlari, M.: Detection of Keratoconus and Suspect Keratoconus by Machine Vision, Hong Kong 3 (2012).

[22]Ruiz Hidalgo, I., et al.: Evaluation of a machine-learning classifier for keratoconus detection based on scheimpflug tomography. Cornea 35, 827-832 (2016).

[23]Jmour, N., Zayen, S., Abdelkrim, A.: Convolutional neural networks for image classification. In: 2018 International Conference on Advanced Systems and Electric Technologies (IC_ASET), pp. 397-402. IEEE (2018).

[24] Lavric, A., Valentin, P.: KeratoDetect: keratoconus detection algorithm using convolutional neural networks. Comput. Intell. Neurosci. 2019, 1-9 (2019).

[25] Lin, S.R., Ladas, J.G., Bahadur, G.G., Al-Hashimi, S., Pineda, R.: A review of machine learning techniques for keratoconus detection and refractive surgery screening. Semin. Ophthalmol. 34, 317-326 (2019).

[26] Perry, H.D., Buxton, J.N., Fine, B.S.: Round and oval cones in keratoconus. Ophthalmology 87, 905-909 (1980)

[27] Ishii, R., et al.: Correlation of corneal elevation with severity of keratoconus by means of anterior and posterior topographic analysis. Cornea 31, 253-258 (2012).

[28]J. Platt, "Fast training of support vector machines using sequential minimal optimization," in Advances in Kernel Methods: Support Vector Learning, C. Scholkopf, C. Burges, and A. Smola, Eds., MIT Press, Cambridge, MA, USA, 1998. [29]D.Anderson, "Understanding the corneal topography, Center, vol. 1, pp. 1-12, 2000.

[30] J. J. Rozema, P. Rodriguez, I. Ruiz Hidalgo, R. Navarro, "SyntEyes KTC: higher order statistical eye model for developing keratoconus," Ophthalmic and Physiological Optics, vol. 37, no. 3, pp. 358-365, 2017. 